\title{
Stärkung der Aktionärsrechte
}

\author{
Diskussionsbeitrag aus der Sicht der schweizerischen Praxis und Aktienrechtslehre
}

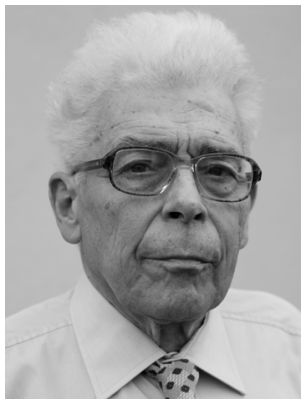

\section{Max Boemle}

Aktienrechtliche Grundkonzepte, Erscheinungsformen der Publikumsaktiengesellschaft, Aktionärsstrukturen, institutionelle Anleger

Basic concepts of corporate law, forms of the public limited company, shareholder structures, institutional investors

Der Beitrag von Egon Franck (Die Unternehmung, Heft 3/2011) empfiehlt eine Rückbesinnung auf die Board Primacy im Hinblick auf die anstehende Aktienrechtsrevision. Aus der Sicht der schweizerischen Aktienrechtspraxis werden zusätzliche Betrachtungen zu seinen Vorschlägen angestellt, gestützt auf die aktienrechtlichen Grundkonzepte und eine aktuelle Analyse der Aktionärsstrukturen von börsenkotierten schweizerischen Publikumsgesellschaften.

The article by Egon Franck (Die Unternehmung, Vol. 65, No. 3) recommends a return to board primacy in view of the imminent revision of corporate law. Further observations concerning his proposals are presented from the standpoint of the practice of Swiss corporate law, based on the fundamental concepts of corporate law and a current analysis of the shareholder structures of listed Swiss public companies.

\section{Entwicklung der aktienrechtlichen Grundkonzepte}

Der stetige Wandel des wirtschaftlichen und gesellschaftlichen Umfelds verlangt in kürzeren Zeitabständen die Anpassung der Rechtsordnung an die Rechtswirklichkeit. Es ist deshalb erstaunlich, dass eine vertiefte Diskussion um eine Erneuerung des schweizerischen Aktienrechts von 1936 erst Ende der 1950er Jahre eingesetzt hat. Das Grundkonzept des Aktienrechts von 1936 ist ein Paradebeispiel der Board Primacy. ${ }^{1}$ Durch eine Stärkung der Macht der Verwaltung soll das „Unternehmen an sich“ geschützt und dessen Fortbestand gesichert werden. Die ersten Revisionspostulate betrafen jedoch keine aktienrechtlichen Schwerpunkte. Mit dem Ziel der breiten Eigentumsstreuung wurden 1961 Massnahmen zur Popularisierung des Aktienbesitzes durch Schaffung von Kleinaktien ${ }^{2}$ verlangt. $^{3}$ „Das dauernde Gedeihen“ (aOR 671) setzt voraus, dass die Verwaltung von den wechselnden Mehrheiten in der Generalversammlung möglichst unabhängig entscheiden kann. Das Ak-

1 Die in der schweizerischen Aktienrechtslehre nicht übliche Bezeichnung wird wegen ihrer Prägnanz von Franck (2011) übernommen.

2 Kleinaktien und nennwertlose Aktien.

3 Bericht der vom Eidg. Volkswirtschaftsdepartement (EVD) eingesetzten Studiengruppe (1961). Die Experten lehnten die Forderungen von Kleinaktien durch den Gesetzgeber ab, nicht zuletzt auch deshalb, weil eine breite Streuung des Aktienbesitzes die Bildung von dauernden Mehrheiten und damit eine langfristige stabile Unternehmenspolitik erschwere. 
tienrecht 1936 hat deshalb das Recht auf Mitwirkung der Aktionäre eingeschränkt. Die Interessen der Publikumsaktionäre, welche Aktien zur gewinnbringenden Anlage oder damals eher selten - zu Spekulationszwecken erworben haben, konnten weitgehend ignoriert werden, wie Hug (1934) anschaulich dargestellt hat. Die Generalversammlungen der grossen Gesellschaften waren denn auch bis weit in die 1970er Jahre eine blosse Formalität.

Auf ein Gegengewicht in der Form einer umfassenden Rechenschaftspflicht der Verwaltung, sowie von Schutzrechten des einzelnen Aktionärs, wie dies bei der Beratung des Aktienrechts von wissenschaftlicher Seite gefordert wurde, hat der Gesetzgeber 1936 bewusst verzichtet. Dieses Grundkonzept des Aktienrechts blieb bis in die 1960er Jahre unbestritten. Die dem Verwaltungsrat zustehenden Freiräume zur vielfach selbstherrlichen Leitung der Aktiengesellschaft stiessen erst unter dem Einfluss der Rechtsentwicklung im Ausland, der zunehmend breiteren Streuung des Aktienbesitzes und dem Auftreten von Finanzinvestoren als aktive Aktionäre auf wachsende Opposition.

$\mathrm{Zu}$ einem kraftvollen Kampf gegen die den schweizerischen demokratischen Überlieferungen widersprechende Machtfülle der Verwaltung setzte als erster Gautschi (1966) in seinem amtlichen Gutachten über die Revisionsbedürftigkeit des Aktienrechts an.

Mit der Gesetzesrevision 1991 wurden die Mängel des Board Primary Board-Konzept der Aktienrechtsordnung 1936 durch eine massvolle Verschärfung der Rechnungslegungsvorschriften und die Verbesserungen zum Schutz der Aktionärsvermögensrechte (Vinkulierung, Bezugsrecht) wenigstens teilweise behoben. Ein wesentliches Merkmal des Aktienrechtes 1991 ist das Festhalten am Boardsystem (Verwaltungsratssystem) und an der zentralen Entscheidungskompetenz durch die Umschreibung der unübertragbaren Hauptaufgaben des Verwaltungsrats. Das schweizerische Aktienrecht bleibt gesellschaftsfreundlich. ${ }^{4}$ Aktionäre, die sich gegen den Verwaltungsrat wehren wollen, haben es nicht leicht, weil sich die Gerichte scheuen, in Gesellschaftsangelegenheiten einzugreifen und beispielsweise zum Schutz von Minderheitsaktionären Generalversammlungsbeschlüsse aufzuheben (Botschaft 1983). Nicht nur das Gesetz, sondern auch die Rechtsprechung ist ausgesprochen verwaltungsratsfreundlich.

Das gegenteilige Grundkonzept einer Aktiendemokratie mit einer konsequenten Gleichberechtigung der Aktionäre bezüglich der Stimmkraft (one share one vote) ist und bleibt wohl in der Schweiz ein theoretisches Idealmodell. Die Macht des Verwaltungsrats verstärkende Stimmrechtsaktien (OR 693), statutarische Stimmrechtsbeschränkungen sowie Vinkulierungsvorschriften sind nach wie vor stark verbreitet. Die Bildung einer Opposition an Generalversammlungen wird überdies durch die problematische Bestimmung zur „gemeinsamen Absprache " sogenannter organisierter Gruppen erschwert. ${ }^{5}$

Die Aktienrechtsordnung ist eine Schnittstelle zwischen Recht und Betriebswirtschaft. Die von aktienrechtlichen Lehrmeinungen schon in der Zwischenkriegszeit vertretene These vom „Unternehmen an sich“, verkörpert im schweizerischen Recht in der Verpflichtung der Verwaltung auf „das dauernde Gedeihen des Unternehmens“ (OR 669 III), wurde in der Folge auch durch die Betriebswirtschaftslehre gestützt (Walther 1959; Ulrich 1990). Die ausgeprägte Selbstfinanzierung machte die schweizerischen Gesellschaften während

4 Vgl. Mendiardi, in: Aktienrechtsreform $(1984,36)$.

5 Vgl. Böckli (2010, 794) und besonders kritisch Spillmann (2004, 187). Mit den Gruppenklauseln will der Verwaltungsrat die Umgehung von Stimmrechtsbeschränkungen bei Generalversammlungsbeschlüssen verhindern. 
Jahrzehnten weitgehend von den Aktionären und vom Finanzmarkt unabhängig, weshalb der Börsenwert vernachlässigt werden konnte. Erst unter dem Einfluss der ShareholderValue-Ausrichtung (Volkart 1998; Schiltknecht 2004) wurde für börsenkotierte Gesellschaften die Erhöhung des Börsenwertes der Gesellschaft zu einem selbständigen Unternehmensziel, weshalb alle Massnahmen geboten wären, welche den Wert der Aktien steigern (Schluep 2003). Daraus leitet sich die Forderung auf Stärkung der Rechte der Aktionäre als Eigentümer ab. ${ }^{6}$

\section{Erscheinungsformen der Aktiengesellschaft}

Die Rechtswirklichkeit zeigt, dass es verschiedene Aktiengesellschaftstypen und Aktionärskategorien gibt. Die einheitlich gedachte Aktiengesellschaft wird für recht unterschiedliche Gebilde verwendet. Sie dient der börsenkotierten Gross-AG, zahlreichen KMU als Ein-Personen- oder Familien-AG, in Konzernen sowohl als Dachgesellschaft und als Ein-Personen-Tochter-AG, ebenso wie gemischtwirtschaftlichen und der reinen öffentlichen Aktiengesellschaft privaten Rechts als Rechtskleid (Druey 2010). Der Bundesrat hielt bei der Aktienrevision in der Botschaft $1983^{7}$ jedoch ausdrücklich am Grundsatz der Einheit des Aktienrechts fest.

Erst mit dem BEHG 1993 ist durch die Hintertür die Einheit des Aktienrechts weitgehend aufgehoben worden. Börsenkotierte Gesellschaften haben zahlreiche besondere Vorschriften zu beachten.

Wegen Schwierigkeiten bei der Festlegung von Abgrenzungskriterien zwischen Kleinund Grossgesellschaften verzichtet allerdings auch der Entwurf 2007 (Botschaft 2007) zur Änderung des Aktienrechts auf eine formelle Zweiteilung. Materiell erfolgt jedoch eine klare Differenzierung.

Der Begriff der Publikumsgesellschaft wurde mit der Neuordnung des Revisionsrechtes $2007^{8}$ ins schweizerische Gesellschaftsrecht eingeführt (OR 727). Als solche gelten Gesellschaften mit kotierten Beteiligungspapieren und ausstehenden Anleihensobligationen. Die Begriffe börsenkotierte Gesellschaft und Publikumsgesellschaft werden in der Folge synonym verwendet.

\section{Realtypen von Publikumsgesellschaft}

Unter den rund 190.000 Ende 2010 im Handelsregister eingetragenen Aktiengesellschaften entspricht nur eine verschwindend kleine Minderheit der gesetzlichen Legaldefinition einer Publikumsgesellschaft. Wird eine Stärkung der Aktionärsrechte gefordert, ist diese Kategorie von Aktiengesellschaften betroffen.

6 Die Feststellung von Egon Franck, die Aktionäre seien keine Eigentümer der Aktiengesellschaft ist sachlich korrekt. Sie wird durch eine Studie von Planta „Sind die Aktionäre auch aus schweizerischer Sicht wirklich Eigentümer der Gesellschaft? " in Recht und Wirtschaft in der Praxis, Festgabe für Rudolf Tschäni (Zürich/St. Gallen 2010, 397 ff.) ausdrücklich bestätigt. „Die Mitgliedschaftsposition der Aktionäre hat nichts mit dem sachenrechtlichen Eigentum zu tun." Hinter dem formellen Eigentum der Gesellschaft steht materiell allerdings „Haben und Nutzen der Aktionäre“. Vgl. Schluep, Mitbestimmung: Bemerkungen zum Verhältnis von Aktiengesellschaft, Unternehmen und öffentlichem Interesse, in: Festschrift Bürgi (1971, 324).

7 Er hält am Prinzip der Einheit des Aktienrechtes fest und nimmt nur in wenigen Einzelpunkten eine differenzierte Regelung vor.

8 Änderung von OR $727-731$ b (in Kraft seit 1.1.2008). 
Bei den Publikumsaktiengesellschaften hat die Zusammensetzung des Aktionariates eine zentrale Bedeutung für die Gestaltung und Ausübung der Aktionärsrechte. Bedauerlicherweise ist die Transparenz zu den Aktionärsstrukturen, abgesehen von den börsenrechtlichen Meldepflichten, bei der Grosszahl schweizerischer Publikumsgesellschaften unbefriedigend. Angaben über die Entwicklung über einen längeren Zeitabschnitt fehlen vollständig.

Gestützt auf eine Analyse der Aktionärsstrukturen von 212 börsenkotierten schweizerischen Aktiengesellschaften ${ }^{9}$ können folgende Realtypen von Publikumsgesellschaften unterschieden werden.

\section{1. unechte Publikumsgesellschaften (82)}

Bei Gesellschaften mit einem Mehrheitsaktionär kann sich dieser auf Kapital- und Stimmenmehrheit (Beispiele: Barry Callebaut, Ems-Chemie) oder dank Stimmrechtsaktien oder Partizipationsscheinen (Beispiele: Kudelski, Schindler) ausschliesslich auf die Stimmenmehrheit abstützen. Für die Zulassung zur Kotierung an der SIX ist ein Free Float von mindestens 25\% vorgeschrieben. $75 \%$ der Aktien können somit auch bei Börsenkotierung im festen Besitz von Grossaktionären liegen.

\subsection{Publikumsgesellschaften mit qualifizierten Beteiligten (101)}

Als qualifizierte Beteiligung bezeichnet das Bankengesetz (Art. 3, Abs. 2, $\mathrm{c}^{\text {bis}) ~ B e t e i l i g u n g e n ~}$ von $10 \%$ des Kapitals oder der Stimmen. Diese ermöglicht einem Grossaktionär einen nicht zu unterschätzenden Einfluss auf die Unternehmungspolitik (z. B. FusG Art. 18 Abs. 5).

\subsection{Publikumsgesellschaften mit mehreren bedeutenden Aktionären (24)}

Als bedeutende Aktionäre umschreibt OR 663 c solche, deren Beteiligung 5\% aller Stimmrechte übersteigt. Für börsenkotierte Gesellschaften ist der tiefste Schwellenwert einer meldepflichtigen Beteiligung mit BEHG 20, Abs. 1 auf 3\% gesenkt worden. Bei Publikumsgesellschaften mit mehreren meldepflichtigen, aber nicht qualifizierten Aktionären könnten diese in gemeinsamer Absprache mit anderen bedeutenden Aktionären Abstimmungsverhältnisse beeinflussen, was jedoch - wie erwähnt - nicht einfach zu realisieren ist.

\subsection{Klassische Publikumsgesellschaften (5)}

Diese zeichnen sich durch ein atomisiertes Aktionariat aus und weisen höchstens einen Aktionär mit einer die börsenrechtliche Meldepflicht nicht wesentlich übersteigenden Anzahl Aktien aus. Mit 3-5\% der Stimmen können die Beschlüsse einer Generalversammlung auch bei der üblichen tiefen Präsenz unter 50\% der stimmberechtigten Aktien nicht beeinflusst werden.

\section{Folgen der Diskrepanz zwischen Rechtswirklichkeit und Corporate Governance}

Egon Franck fordert an der Board Primacy mit einem als Treuhänder für alle Shareholders und Stakeholders tätigen Verwaltungsrat festzuhalten, der zur Vermeidung von Interessen-

9 Erstellt gestützt auf den Aktienführer Schweiz 2012 (Verlag Finanz und Wirtschaft, 2011). 
konflikten weder Aktien noch Optionen halten darf. Wird dieser Vorschlag der Rechtswirklichkeit schweizerischer börsenkotierter Aktiengesellschaften gerecht? Bei den 82 unechten Publikumsgesellschaften dürfte er nicht umsetzbar sein, weil die Mehrheitsaktionäre nicht bereit sein werden, auf ihre Entscheidungs- und Kontrollkompetenzen zu verzichten und trotzdem das volle Unternehmerkapitalrisiko zu tragen. Bei diesem Gesellschaftstyp steht für den Gesetzgeber der generelle Minderheitenschutz im Vordergrund.

Anders liegen die Verhältnisse bei den drei übrigen Typen von Publikumsgesellschaften. Bei den Bestrebungen zur Stärkung der Aktionärsrechte kann jedoch eine Erscheinung nicht übersehen werden, nämlich die weitverbreitete Indolenz von Publikumsaktionären (Amstutz 2007). Viele fühlen sich nicht als Mitglieder einer Körperschaft und beteiligen sich daher auch nicht an der Willensbildung und Kontrolle durch Teilnahme an einer Generalversammlung. Ökonomisch besteht zwischen ihnen und den Investoren in Anlagefonds nach KAG Art. 25 kein Unterschied. Die Gleichgültigkeit von Investoren gegenüber Mitwirkungsrechten wird besonders deutlich durch die Dispoaktionäre veranschaulicht, welche auf den Eintrag im Aktienbuch und damit auf die Mitwirkungsrechte überhaupt verzichten. Sie sind ausschliesslich an der Rendite ihres meist kurzfristigen Aktieninvestments interessiert. Einen Gegensatz verkörpern Finanzinvestoren, welche sich offen zum Aktionärsaktivismus bekennen oder diesen für die übrigen Aktionäre oft in unerwünschter Weise hinter den Kulissen ausüben. Für dieses Shareholder Sharking sind auch in der Schweiz Beispiele bekannt. Zu wenig beachtet wird im Recht der Publikumsgesellschaft die wachsende Bedeutung der institutionellen Anleger (Spillmann 2004) aus dem Kreis der Vorsorgeeinrichtungen und Versicherungen, welche mit ihrem Aktienbesitz breite Bevölkerungskreise in Beziehung zu den Aktienmärkten und indirekt zu den Aktionärsrechten bringen. Offen ist auch die Frage, mit welchen Anreizen bedeutende Aktionäre für eine längere Zeitspanne an die Gesellschaft gebunden werden könnten, um damit ein Gegengewicht zu den kurzfristigen Aktientradern zu bilden. Es wäre deshalb nicht nur zum Verhältnis Board/Aktionäre, sondern auch zu jenem der verschiedenen Aktionärskategorien unter sich, eine vertiefte wissenschaftliche Diskussion erwünscht.

\section{Literaturhinweise}

Aktienrechtsreform (1984): Schriftenreihe der Schweizerischen Treuhand- und Revisionskammer, Zürich.

Amstutz, M.D. (2007): Macht und Ohnmacht des Aktionärs, Zürich.

Birchler, U., et al. (2011): Aktienbesitz in der Schweiz, Institut für Banking and Finance, Universität Zürich.

Böckli, P. (2009): Schweizer Aktienrecht, 4. Aufl., Zürich.

Botschaft über die Revision des Aktienrechts vom 23.2.1983, S. 2.

Botschaft zur Änderung des Obligationenrechts vom 21.12.2007, S. 1606.

Bürgi, W.F. (1971): Festgabe zum 70. Geburtstag, Lebendiges Aktienrecht, Beitrag von W.R. Schluep: Mitbestimmung, Zürich.

Druey, J.N., et al. (2010): Gesellschafts- und Handelsrecht, Zürich.

Forstmoser, P., et al. (2003): Festschrift zum 60. Geburtstag: Neuere Tendenzen im Gesellschaftsrecht, Beitrag von W.R. Schluep: Vom „Unternehmen an sich“ über „Corporate Governance“ bis zum „Bond Value“ - so what?, Zürich. 
Gautschi, G. (1966) (unter Mitwirkung von Käfer, K.): Bericht und Vorschläge zu einer Revision des schweizerischen Aktienrechts (unveröffentlichtes Manuskript an das Eidg. Justiz- und Polizeidepartement Zürich.

Hug, W. (1934): Zur Revision des schweizerischen Aktienrechts, S. 29.

Meier-Hayoz, A./Forstmoser, P. (2007): Schweizerisches Gesellschaftsrecht, 10. Aufl., Bern.

Schiltknecht, K. (2004): Corporate Governance, Zürich.

Spillmann, T. (2003): Institutionelle Investoren im Recht der (echten) Publikumsgesellschaft, Zürich.

Tschäni, R. (2009): Festgabe zum 60. Geburtstag, M\&A: Recht und Wissenschaft in der Praxis, Zürich.

Ulrich, H. (1990): Unternehmungspolitik, Bern, S. 103.

Volkart, R. (1998): Shareholder Value \& Corporate Valuation, Zürich.

Walther, A. (1959): Einführung in die Wirtschaftslehre der Unternehmung, 1. Band, Zürich, S. 9.

Max Boemle, Dr. oec., Emeritierter Professor für Finanzmanagement und Financial Accounting an der Universität Freiburg, Honorarprofessor für Financial Accounting an der Universität Lausanne.

Anschrift: Mohnstrasse 108, CH-3084 Wabern, Tel.: +41 (0)31/964-22-05, Fax: +41 (0)31/964-22-06, Email: max.boemle@bluewin.ch 